subtypes, specialized software named Protein Reader with implemented Dust algorithm have searched through the NCBI $\mathrm{nr}$ database, that contains the records of more than $400 \mathrm{E}$ coli subtypes. The median age of patients was 7.14 and 7.11, respectively.

Various E coli subtypes (P0301867.1-10, O104:H4, O103: $\mathrm{H} 25$, O111:H11, KTE and K) were three times more abundant in patients with JIA, while in children with ReA, the abundance of diarrheagenic E. coli (DEC) was detected.

Many studies have speculated the influence of gut microbiota in the development of arthritis in children. Despite the technological advancements in the examination of microbiota composition, there are still many limitations imposed by patient selection, methodology and data analysis.

Besides, without the proper definition of 'healthy' microbiome as a reference standard, it is challenging to distinguish alterations responsible for diseases development. In our proofof-the-concept study microbiota was therefore compared in two groups of patients both presenting with arthritis.

Since $\mathrm{E}$ coli is one of the paramount bacteria in gut microbiota with more than 600 recognized subtypes, it is reasonable to assume that described differences can have a potential impact on the gut environment, with the contribution to the development of the chronic disease in JIA patients or the resolution of symptoms in children with ReA. While this observation needs conformation in multiple time points and larger patient cohorts, it pinpoints gut microbiota as a potential new therapeutic target in the treatment of chronic inflammatory diseases.

\section{LONG TERM FOLLOW-UP OF THE PATIENTS WITH ANTI NUCLEAR ANTIBODY POSITIVITY WHO HAD INITIALLY NO IDENTIFIABLE RHEUMATIC DISEASES}

Ilayda Altun*, Mehmet Yildiz, Gizem Yilmaz, Ayten Aliyeva, Fatih Haslak, Oya Koker, Amra Adrovic, Sezgin Sahin, Kenan Barut, Ozgur Kasapcopur. Istanbul UniversityCerrahpasa, Pediatric Rheumatology, Istanbul, Turkey

10.1136/archdischild-2021-europaediatrics.433

Anti-nuclear antibodies (ANA) are a group of the antibodies that develop against intracellular components of the cells. It is usually useful for diagnosing some of the connective tissue diseases like systemic lupus erythematosus, mixed connective tissue disease.

But it is reported that its positivity rate is about in healthy individuals.

Therefore, it can be confusing to check ANA test, if there is not really high suspicion for connective tissue diseases or juvenile idiopathic arthritis.

We aimed to evaluate results of long-term follow-up of the patients with ANA positivity who had initially no identifiable rheumatic diseases.

Six hundred and ninety-four patients with ANA positivity who did not diagnosed as any of the rheumatic diseases at the first examination were found in database. Two hundred and eightytwo patients of them were called so far and questioned about their demographic features and symptoms that are related with rheumatic diseases.

Mean age of the patients at the time of study and at the time of testing were $13.4 \pm 4.5$ and $9.1 \pm 4.0$ years. The female: male ratio was 1.05. Mean follow-up duration was
$4.3 \pm 2.8$ years. Most common reasons for the request for ANA test were arthralgia (n:99 (D.1)) and skin eruptions (n: 54 (24.1)). ANA testing was most commonly requested by a general pediatrists.

Most of the diseases(Hypermobility Syndrome, Urticaria, Hypothyroidism, Transient synovitis, Idiopathic Thrombocytopenic Purpura, Scoliosis) were diagnosed in patients with ANA positivity were not related with autoimmune mechanisms that associated with ANA positivity therefore, these diseases are thought to be coincidence. Only in 1 patients, systemic lupus erythematosus that has certain association with ANA positivity were diagnosed.

We are reporting that in only $0.3 \%$ of patients with ANA positivity who don't have any diseases diagnosed initially, were diagnosed as rheumatologic diseases during to the follow-up period.

Since positivity of ANA is also common in the healthy population, requesting this test in only patients with high suspicion for connective tissue disease will reduce confusion in terms of diagnosis.

\section{CASTLEMAN DISEASE PRESENTED WITH PROLONGED FEVER OF UNKNOWN ORIGIN}

Tamara Janjić* ${ }^{*}$ M Pavlović, L Lamot, J Stepan, M Harjaček, M Vidović. Sestre milosrdnice University Hospital Center

10.1136/archdischild-2021-europaediatrics.434

Castleman disease presented with prolonged fever of unknown origin- a case report Janjic $\mathrm{T}(1)$, Pavlovic M(2), Lamot L(3), Stepan J(2), Harjacek M(3), Vidovic M(3)

(1) Sestre milosrdnice University Hospital Center

(2) Children's Hospital Zagreb, Department of Oncology and Hematology

(3) Sestre milosrdnice University Hospital Center, Department of Pediatrics, Division of clinical immunology and rheumatology

Fever is the most common cause for which children and their caregivers seek medical attention. If accompanied by elevated blood inflammatory markers, infectious diseases are suspected. However, if microbiological findings are negative, and if there is no response to antimicrobial therapy, more careful and comprehensive evaluation is required. Amongst many disorders, one of the rare but often misdiagnosed cause is Castleman disease (CD). This is a heterogeneous group of lymphoproliferative disorders with similar histopathologic features divided into three types. Unicentric CD involves one or more enlarged lymph node(s) in a single region of a body while multicentric Castleman disease involves multiple regions of lymphadenopathy and is further subclassified in HHV-8 positive and HHV-8 negative/idiopathic type.

We present a case of a 4.5 -year-old boy who came to our pediatric emergency department with a history of intermittent fever for almost a month, cough, skin rash with spontaneous regression, fatigue, periodic leg pain, night sweats, and weight loss. The physical examination showed few mildly enlarged cervical lymph nodes, pale skin, antalgic gait and fever. Routine blood tests revealed elevated C-reactive protein level and erythrocyte sedimentation rate, anemia, and thrombocytosis. He was admitted to a Pediatric Rheumatology department for evaluation. Other laboratory tests showed elevated fibrinogen, 